\title{
FUNDAMENTAL SYSTEM OF EQUATIONS FOR MOMENTUM AND ENERGY OF ELECTROMAGNETIC FIELD IN INHOMOGENEOUS MEDIUM
}

\section{Arthur A. Dyshekov}

Berbekov Kabardino-Balkarian State University, http://www.kbsu.ru

Nalchik 360004, Russian Federation

dyshekov@yandex.ru

\begin{abstract}
An alternative approach to the description of the interaction of the electromagnetic field with the crystal is proposed, in which the main characteristics are the energy and pulse densities. The reaction of the medium to the external perturbation is considered as a local change in geometry - the rotation of the orthogonal basis built on the induction vectors and the pulse of the field, determined by the structural characteristics of the medium. The equations that allow calculating the momentum and energy of the field in its interaction with the crystal are obtained.
\end{abstract}

Keywords: Energy-momentum tensor, electromagnetic field, Maxwell tensor, Gâteaux derivative, canonical form of the field tensor

PACS: 61.05.C

Biblography - 16 references

Received 13.06.18, accepted 13.01.19

RENSIT, 2019, 11(2):103-112

DOI: $10.17725 /$ rensit.2019.11.103

CONTENTS

1. INTRODUCTION (103)

2. LAWS TO CHANGE THE MOMENTUM AND ENERGY OF THE FIELD (104)

3. The canonical form of the Maxwell tensor AND CONDITIONS FOR THE FIELDS (105)

4. Relativistic substantiation (106)

5. Fundamental equations in the local basis (107)

6. Conclusion of DifFerential operations $\nabla^{\prime}$ $\cdot \mathbf{P}, \nabla^{\prime} \cdot \mathrm{T}, \nabla^{\prime} \cdot \varepsilon(108)$

6.1. Calculation $\nabla^{\prime} \cdot \mathbf{P}(108)$

6.2. Calculation $\nabla^{\prime} \cdot \varepsilon(109)$

6.3. Calculation $\nabla^{\prime} \cdot \mathrm{T}(109)$

7. The Calculation of the Rotation Vector $\varphi$ (109)

8. Conclusion of the basic equation System in THe Cartesian basis (110)

9. Conclusion (110)

REFERENCES (111)

\section{INTRODUCTION}

A new approach to describing the interaction of the electromagnetic field with a substance based on the density of energy and momentum is proposed. Known approaches for describing wave fields in matter are based on the analysis of Maxwell's equations with respect to inductions [1-4]. However in the experiment field strengths are not measured for obvious reasons. In the experiment, the wave intensity is measured, and the direction of wave propagation, the Umov-Poynting vector, is associated with the wave momentum. Compliance with experiment is achieved by calculating these characteristics through field strengths. For example, to find the reflection coefficient of a wave from a medium, it is necessary to calculate the Pointing vector component, normal with respect to the surface.

Momentum and energy (or rather, their densities) are quadratic in fields. As a result they cannot be directly represented as a superposition of the resulting field solutions. The answer is only in the final stage, when the solutions for the fields have already been received. On the one hand, this is a definite plus (it is much easier to solve linear equations than nonlinear ones). On the other hand, such a procedure makes it difficult to directly analyze the intensity distribution in a given direction of space, depending on the characteristics of the scattering object.

In this regard we can formulate the following problem statement. Describe the interaction of the field and matter by means of the energy and momentum densities in accordance with the equations of motion of the electromagnetic field. 
As is known, the equations of motion of a dynamical system (the Euler-Lagrange equations) are obtained from the variational principle as a condition for the extremality of a certain action functional. This principle is valid both for mechanical systems and for fields - systems with an infinite number of degrees of freedom. In this case, the invariance of an action with respect to a certain group of coordinate transformations (external symmetries) or gauge transformations (internal symmetries) leads to covariant equations of motion and to fundamental conservation laws.

The conservation laws follow from the first theorem of Noether [5-7] which is formulated for global and local symmetry transformations of the action functional. The meaning of this theorem is as follows. If the action is invariant with respect to transformations of global symmetries that form a certain Lie group [7] then for each symmetry transformation and any solution of the Euler-Lagrange equations, the quantities called currents are saved. Keeping current means that its 4-divergence is zero. Global invariance means that the transformation parameter is independent of space-time points. In turn the integration of currents over specially selected areas of space-time gives the remaining charges corresponding to each current.

Let the action be invariant with respect to translations in space-time. Then the general expression for the current passes into the expression for the energy-momentum tensor for fields whose 4-divergence turns to zero by virtue of the first Noether theorem. It is the 4-divergence of the energy-momentum tensor that describes the electromagnetic field as a dynamic system.

Note that the first Noether theorem is formulated for infinitesimal space-time translations. The Lie group in the formulation of the first Noether theorem [6] is directly related to the continuity of space-time symmetries. This circumstance is of decisive importance in the future case of the X-ray wavelength range.

Since for an $\mathrm{X}$-ray wave, the dielectric constant is a function of coordinates, the continuity of translations on atomic scales is incompatible with the condition of medium homogeneity. Only in the particular case of the crystalline medium is the invariance of the action functional ensured and only with respect to the discrete and not continuous, group of spatial translations on the Bravais lattice vectors. Thus the direct application of the first theorem of Noether to construct the energy-momentum tensor as the dynamic basis of the theory taking into account the microscopic structure of the medium, is impossible.

\section{LAWS TO CHANGE THE MOMENTUM AND ENERGY OF THE FIELD}

In connection with the above, a different approach is proposed, which allows us to obtain the relations of interest directly from the equations of classical macroscopic electrodynamics.

We proceed from the Maxwell equations for the field in the absence of charges and currents in the medium:

$$
\begin{aligned}
& \nabla \times \mathbf{E}=-\frac{1}{c} \frac{\partial \mathbf{H}}{\partial t} \\
& \nabla \times \mathbf{H}=\frac{1}{c} \frac{\partial \mathbf{D}}{\partial t} .
\end{aligned}
$$

For the X-ray wavelength range the permittivity $\varepsilon$ and hence the polarizability $\chi$ is a function of the coordinates:

$$
\mathbf{D}=\varepsilon(\mathbf{r}) \mathbf{E}=(1+\chi(\mathbf{r})) \mathbf{E} .
$$

In addition the interaction of electromagnetic waves with the medium and in other ranges can be described by the formula (2). For example artificial periodic structures - photonic crystals [8], objects of X-ray optics for soft X-rays [9] also formally correspond to (2) since the elements scattering radiation form a certain spatial structure.

In formulas (1), (2) and further standard notation is used, $\nabla-$ Hamilton operator in some Cartesian basis $\mathbf{i}_{k}$, summation is performed on the mute index k.

Let us give a generalization to the case of $\varepsilon=$ $\varepsilon(\mathrm{r})$ of the well-known in electrodynamics of the derivation of the law of momentum variation [10]. Consider the value $(\nabla \times \mathbf{E}) \times \mathbf{D}$ in some orthonormal cartesian basis $\mathbf{i}_{\mathrm{m}}$ :

$$
\begin{aligned}
& (\nabla \times \mathbf{E}) \times \mathbf{D}=\nabla \cdot(\mathbf{D E})- \\
& -\frac{1}{2} \nabla \cdot(\mathbf{I} \cdot(\mathbf{D} \cdot \mathbf{E}))+\frac{E^{2}}{2} \nabla \varepsilon .
\end{aligned}
$$

Similarly we find the value $(\nabla \times \mathbf{H}) \times \mathbf{H}$ : 


$$
(\nabla \times \mathbf{H}) \times \mathbf{H}=\nabla \cdot(\mathbf{H H})-\frac{1}{2} \nabla \cdot\left(\mathbf{I} \cdot H^{2}\right) .
$$

In (3) and (4) the values $\mathbf{D E}=D_{i} E_{j} ; \mathbf{H H}=$ $H_{i} H_{\text {, }}$, representing dyads (external products) [11] as well as the unit tensor $\mathbf{I}=\mathbf{i}_{\mathrm{m}} \mathbf{i}_{\mathrm{m}}$. In the sequel an expression of the form $\mathbf{a b}$ will mean the outer product of vectors a and $\mathbf{b}$. In deriving (3) and (4), it was taken into account that according to Maxwell's equations in the absence of charges $(\nabla \cdot \mathbf{D})=0$ and $(\nabla \cdot \mathbf{H})=0$. Note that due to the invariance of the operator $\nabla$, formulas (3) and (4) are valid in any basis.

Find the sum of (3) and (4) and, taking into account (1), after a series of transformations we get

$\nabla \cdot\left(\mathbf{D E}+\mathbf{H H}-\frac{1}{2} \mathbf{I} \cdot\left(\mathbf{D} \cdot \mathbf{E}+H^{2}\right)\right)+$

$+\frac{E^{2}}{2} \nabla \varepsilon=\frac{1}{c} \frac{\partial}{\partial t}(\mathbf{D} \times \mathbf{H})$.

Imagine this equation as

$$
\nabla \cdot \mathbf{T}+\frac{E^{2}}{2} \nabla \varepsilon=\frac{\partial \mathbf{P}}{\partial t} \text {. }
$$

The following values are entered here: Maxwell stress tensor in medium $\mathrm{T}$

$$
\mathbf{T}=\mathbf{D E}+\mathbf{H H}-\frac{1}{2} \mathbf{I}\left(\mathbf{D} \cdot \mathbf{E}+H^{2}\right)=\mathbf{D E}+\mathbf{H H}-w \mathbf{I} .
$$

energy density

$$
w=\frac{1}{2}\left(\mathbf{D} \cdot \mathbf{E}+H^{2}\right) \text {. }
$$

and also the bulk density of the total momentum of the field $\mathbf{P}=\frac{1}{c}(\mathbf{D} \times \mathbf{H})$ (we will briefly call it the momentum).

As can be seen from (5) the change in the field momentum in the general case is associated not only with the Maxwell tensor $\mathbf{T}$ but also depends on the permittivity gradient $\nabla \varepsilon$. According to (6) taking into account (2) the tensor $\mathbf{T}$ turns out to be symmetric.

Nowform theexpression $\mathbf{H} \cdot(\nabla \times \mathbf{E})-\mathbf{E} \cdot(\nabla \times \mathbf{H})$ and using (1) we get:

$$
\begin{aligned}
& \nabla \cdot(\mathbf{E} \times \mathbf{H})=\mathbf{H} \cdot(\nabla \times \mathbf{E})-\mathbf{E} \cdot(\nabla \times \mathbf{H})= \\
& =-\frac{1}{c} \frac{\partial}{\partial t}(\mathbf{H} \cdot \mathbf{H}+\mathbf{D} \cdot \mathbf{E})=-\frac{1}{c} \frac{\partial w}{\partial t} .
\end{aligned}
$$

Calculate the divergence $c \mathbf{P}$ :

$$
c(\nabla \cdot \mathbf{P})=\varepsilon \nabla \cdot(\mathbf{E} \times \mathbf{H})+\frac{\nabla \varepsilon}{\varepsilon} \cdot(\mathbf{D} \times \mathbf{H}) .
$$

From here

$$
\nabla \cdot(\mathbf{E} \times \mathbf{H})=\frac{1}{\varepsilon} \cdot\left(\nabla \cdot\left(\mathbf{D} \times \mathbf{H}-\frac{\nabla \varepsilon}{\varepsilon^{2}} \cdot(\mathbf{D} \times \mathbf{H})\right) .\right.
$$

Substituting (9) into (8), we have

$\nabla \cdot \mathbf{P}-\frac{\nabla \varepsilon}{\varepsilon} \cdot \mathbf{P}=-\frac{\varepsilon}{c^{2}} \frac{\partial w}{\partial t}$.

\section{THE CANONICAL FORM OF THE MAXWELL TENSOR AND CONDITIONS FOR THE FIELDS}

Equations (5) and (10) form the basic system for momentum and field energy. They generalize the laws of change of momentum and energy of the electromagnetic field in the case $\varepsilon=\varepsilon(\mathbf{r})$. However, the system (5), (10) is generally unclosed with respect to $w$ and $\mathbf{P}$ since the tensor t includes the diads $\mathbf{D E}$ and $\mathbf{H H}$. In addition the change in the momentum according to (5) depends on the amplitude of the electric field through $E^{2} / 2$. Find out what it's connected to and try to shut down the system.

In deriving equations (5) and (10), we used an arbitrary Cartesian basis not related to the electromagnetic field in any way. However it is obvious that the form of the Maxwell tensor $\mathbf{T}$ depends on the choice of the basis in accordance with the transformational properties of the second rank tensor. This circumstance suggests that the dynamic nature of the tensor $\mathbf{T}$ is associated with some selected directions in space, and these directions are determined by the vectors $\mathbf{D}, \mathbf{H}$, and $\mathbf{P}$. We show that this is indeed the case.

The simplest (canonical) form of the tensor $\mathbf{T}$ is in a basis built on eigenvectors. Since the tensor $\mathbf{T}$ is symmetric, its canonical form is a diagonal tensor.

We define the canonical form of the Maxwell tensor (6). To do this we find the eigenvalues $\lambda_{\text {i }}$ and the eigenvectors $\mathbf{e}_{j}$ of the tensor $\mathbf{T}$ in the normalized basis, satisfying the well-known equation:

$\mathbf{T} \cdot \mathbf{e}_{\mathrm{j}}=\lambda \mathbf{e}_{\mathrm{j}}$

The characteristic equation is

$\operatorname{det}(\mathbf{T}-\lambda \mathbf{I})=-\lambda^{3}+I_{1} \lambda^{2}-I_{2} \lambda+I_{3}=0$, where $I_{\mathrm{j}}$ are the main invariants of the tensor $\mathbf{T}$ :

$I_{1}=\operatorname{Tr}(\mathbf{T}), I_{2}=\frac{1}{2}\left(\operatorname{Tr}(\mathbf{T})^{2}-\operatorname{Tr}\left(\mathbf{T}^{2}\right)\right), I_{3}=\operatorname{det}(\mathbf{T})$. 
Here $\operatorname{Tr}(\mathbf{T})$ is the trace of the tensor $\mathbf{T}$. From the form (6) we obtain one eigenvalue $\lambda_{3}=-w$. In fact direct verification ensures that

$$
\begin{aligned}
& \mathbf{T}-\lambda \mathbf{I}=\mathbf{D E}+\mathbf{H} \mathbf{H}-w \mathbf{I}+w \mathbf{I}=\mathbf{D E}+\mathbf{H} \mathbf{H}, \\
& \operatorname{det}(\mathbf{D E}+\mathbf{H} \mathbf{H})=0 .
\end{aligned}
$$

Find the eigenvector $\mathbf{u}_{3}$ corresponding $\lambda_{3}=-w$. Solving (11) for $\lambda_{3}=-w$, we obtain the components of the corresponding vector $\mathbf{u}_{3}$ :

$u_{31}=E_{2} H_{3}-E_{3} H_{2}, u_{32}=E_{3} H_{1}-E_{1} H_{3}, u_{33}=E_{1} H_{2}-E_{2} H_{2}$,

which form a vector

$\mathbf{u}_{3}=\mathbf{u}_{3 \mathbf{j}} \mathbf{e}_{\mathbf{j}}=\mathbf{E} \times \mathbf{H}$.

From here

$$
\mathbf{e}_{3}=\frac{\mathbf{E} \times \mathbf{H}}{|\mathbf{E} \times \mathbf{H}|} \text {. }
$$

We write $\mathbf{T}$ in the orthonormal basis $\mathbf{e}_{i}$ :

$T_{i j}^{\prime}=\mathbf{e}_{i} \cdot \mathbf{T} \cdot \mathbf{e}_{j}$

$$
T_{i j}^{\prime}=\left(\begin{array}{lll}
T_{11} & \mathrm{~T}_{12} & 0 \\
T_{21} & \mathrm{~T}_{22} & 0 \\
0 & 0 & -w
\end{array}\right) .
$$

Now we require that $T_{12}=T_{21}=0$, then, obviously, $T_{11}=\lambda_{1}, T_{22}=\lambda_{2}$, and $\mathbf{e}_{1,2}$ are eigenvectors. We get the conditions:

$\mathbf{T}_{12}=\mathbf{e}_{1} \cdot \mathbf{T} \cdot \mathbf{e}_{2}=\left(\mathbf{e}_{1} \cdot \mathbf{D}\right)\left(\mathbf{E} \cdot \mathbf{e}_{2}\right)+\left(\mathbf{e}_{1} \cdot \mathbf{H}\right)\left(\mathbf{H} \cdot \mathbf{e}_{2}\right)=0$,
$\mathbf{T}_{21}=\mathbf{e}_{2} \cdot \mathbf{T} \cdot \mathbf{e}_{1}=\left(\mathbf{e}_{2} \cdot \mathbf{D}\right)\left(\mathbf{E} \cdot \mathbf{e}_{1}\right)+\left(\mathbf{e}_{2} \cdot \mathbf{H}\right)\left(\mathbf{H} \cdot \mathbf{e}_{1}\right)=0$.

It follows

$\mathbf{e}_{1} \cdot \mathbf{D}=\mathrm{D} ; \mathbf{e}_{1} \cdot \mathbf{E}=\mathrm{E} ; \mathbf{e}_{1} \cdot \mathbf{H}=0$,

$\mathbf{e}_{2} \cdot \mathbf{D}=\mathbf{e}_{2} \cdot \mathbf{E}=0 ; \mathbf{e}_{2} \cdot \mathbf{H}=\mathrm{H}$.

Then

$$
\begin{aligned}
& T_{11}=\mathbf{e}_{1} \cdot \mathbf{T} \cdot \mathbf{e}_{1}=D E-w=\lambda_{1}=\frac{1}{2}\left(D E-H^{2}\right) ; \\
& T_{22}=\mathbf{e}_{2} \cdot \mathbf{T} \cdot \mathbf{e}_{2}=H^{2}-w=\lambda_{2}= \\
& =-\lambda_{1}=\frac{1}{2}\left(H^{2}-D E\right) .
\end{aligned}
$$

And the eigenvectors are equal

$$
\mathbf{e}_{1}=\frac{\mathbf{D}}{D}=\frac{\mathbf{E}}{E} ; \mathbf{e}_{2}=\frac{\mathbf{H}}{H} ; \mathbf{e}_{3}=\frac{\mathbf{P}}{P} \text {. }
$$

Now we require the fulfillment of the additional condition $\lambda_{1}=\lambda_{2}=0$, the justification of which is given below. In this case, an additional bond $D E=$ $\mathrm{H}^{2}$ should be imposed on the fields.

Therefore, the following conditions are necessary for the tensor $\mathrm{T}$ to describe the field:

1) Orthogonality of fields

\section{$\mathbf{D} \cdot \mathbf{H}=\mathbf{D} \cdot \mathbf{H}=0$,}

2) Connection amplitude fields

$D E=\varepsilon E^{2}=H^{2}$.

The existence of the connection of field amplitudes (14) as will be seen further is crucial for the construction of a closed theory. In this case, if $\varepsilon=\varepsilon(\mathbf{r})$ conditions (13) and (14) are realized locally. Also these conditions are for the full field. Thus we obtain a canonical form of the Maxwell tensor $\mathbf{T}$ in the $\mathbf{e}_{\dot{j}}$ basis:

$\mathbf{T}=-w\left(\mathbf{e}_{3} \mathbf{e}_{3}\right)$.

As can be seen the Maxwell tensor $\mathbf{T}$ is locally determined by the energy density $\mathrm{w}$ and the direction of momentum transfer $\mathbf{e}_{3}$. Moreover, in accordance with the theorems on eigenvalues and eigenvectors of the symmetric tensor, the eigenvalues turn out to be real and the eigenvectors corresponding to these values are orthogonal.

\section{RELATIVISTIC SUBSTANTIATION}

To substantiate these conditions, we turn to the results of relativistic electrodynamics. We introduce the four-dimensional tensor of the field $F_{i k}[12]$ in a vacuum in the Minkowski space $R_{1,3}^{4}$. By definition, invariants of the tensor $F_{i k}$ are called the coefficients of the characteristic polynomial

$P(\lambda)=\operatorname{det}\left(F_{i k}-\lambda g_{i k}\right)$,

where gik is the Minkowski metric (+,-,-,-).

Direct calculation leads to the following form of the characteristic polynomial:

$P(\lambda)=-\lambda^{4}+\left(E^{2}-H^{2}\right) \lambda+(\mathbf{E} \cdot \mathbf{H})^{2}$,

whence we get the well-known invariants of the field $E^{2}-H^{2}$ and $\mathbf{E} \cdot \mathbf{H}$.

The question of reducing the skew-symmetric tensor of a field $F_{i k}$ to Lorentz transformations to the canonical form is solved by the following theorem [12].

Theorem 1.

1. Suppose that the invariants of the field $E^{2}-H^{2}$ and $\mathbf{E} \cdot \mathbf{H}$ are not equal to zero.

a) If $\mathbf{E} \cdot \mathbf{H} \neq 0$, then the Lorentz transformation can reduce the tensor $F_{i k}$ to such a form that the vectors $\mathbf{E}$ and $\mathbf{H}$ are parallel and both are nonzero.

b) If $\mathbf{E} \cdot \mathbf{H}=0, E^{2}-H^{2} \neq 0$, then we can reduce the tensor $F_{i k}$ to the form that $\mathbf{E} \neq 0 \mathbf{H}=0$ for $E^{2}-H^{2}$ $>0$ or $\mathbf{E}=0 \mathbf{H} \neq 0$ for $E^{2}-H^{2}<0$.

2. Let $E^{2}-H^{2}=0$ and $\mathbf{E} \cdot \mathbf{H}=0$. Then, after any Lorentz transformation, the vectors $\mathbf{E}$ and $\mathbf{H}$ will 
be mutually perpendicular and equal in length. The tensor $F_{i k}$ can be reduced in this case to the form:

$$
F_{i k}=\left(\begin{array}{llll}
0 & E & 0 & 0 \\
-E & 0 & 0 & E \\
0 & 0 & 0 & 0 \\
0 & -E & 0 & 0
\end{array}\right) \text {. }
$$

We are interested in the second point of this theorem, since it corresponds to a special state of the field, the electromagnetic wave. It is in this case that the condition of coupling the field amplitudes and the transverse condition of the fields arise. As can be seen, condition (14) can be considered as a generalization of the classical invariant of the field $E^{2}-H^{2}$ to the case of electrodynamics of a continuous medium.

Now we justify the condition $\lambda_{1}=\lambda_{2}=0$. As is known, for the tensor of the field $F_{i k}$ with respect to the scalar Lagrangian

$$
L=-\frac{1}{4} F^{i k} F_{i k}
$$

a symmetric four-dimensional energy-momentum tensor $\mathbf{T}$ is constructed. The explicit form of this tensor is determined from the first theorem of Noether [7]. According to the theorem of Noether, since the action for a free electromagnetic field in Minkowski space is invariant with respect to the global action of the Poincaré group (space-time translations plus reflections) there exists a secondrank tensor, whose 4-divergence is zero:

$$
\partial_{k} T_{i}^{k}=0 \text {. }
$$

This quantity, called the current, is the energymomentum tensor:

$$
T_{i}^{k}=\frac{1}{2}\left(-F_{i k} F_{m}^{k}+\frac{1}{4} g_{i k} F^{2}\right) g_{k m} .
$$

Find the eigenvalues of the tensor $T_{i}^{k}$ :

$$
\operatorname{det}\left(T_{i}^{k}-\lambda \delta_{i}^{k}\right)=0 \text {. }
$$

Due to the symmetry of the tensor we obtain four pairwise coinciding eigenvalues:

$$
\begin{aligned}
& 2 \lambda_{1}=2 \lambda_{3}=H^{2}+\frac{1}{2}\left(E^{4}+H^{4}-4(\mathbf{E} \cdot \mathbf{H})^{2}+2 E^{2} H^{2}\right)^{1 / 2}, \\
& 2 \lambda_{2}=2 \lambda_{4}=H^{2}-\frac{1}{2}\left(E^{4}+H^{4}-4(\mathbf{E} \cdot \mathbf{H})^{2}+2 E^{2} H^{2}\right)^{1 / 2} .
\end{aligned}
$$

This shows that if the conditions $E=H, \mathbf{E} \cdot \mathbf{H}=0$ are fulfilled the eigenvalues take the following form:
$\lambda_{1}=\lambda_{3}=E^{2}=w$,

$\lambda_{2}=\lambda_{4}=0$.

Thus, we arrive at the condition that the two eigenvalues of the energy-momentum tensor are zero. Since the Maxwell tensor is the spatial part of the energy-momentum tensor it is clear that this condition must also be satisfied for the Maxwell tensor $\mathbf{T}$ defined by formula (6).

It should be noted that the given justifications used field and energy-momentum tensors in vacuum. The above conclusion for the Maxwell tensor is more General, since we consider the field in the substance.

\section{FUNDAMENTAL EQUATIONS IN THE LOCAL BASIS}

Now, taking into account (14), we can associate the field energy density with the amplitude of the electric vector:

$$
w=\frac{1}{2}\left(\frac{1}{\varepsilon} D^{2}+H^{2}\right)=\frac{E^{2}}{2} \varepsilon(1+\varepsilon) .
$$

From here

$$
\frac{E^{2}}{2}=\frac{w}{\varepsilon(1+\varepsilon)} \text {. }
$$

As a result, equation (5) takes on a compact form:

$$
\nabla \cdot \mathbf{T}+\frac{w}{\varepsilon(1+\varepsilon)} \nabla \varepsilon=\frac{\partial \mathbf{P}}{\partial t} .
$$

Thus we have ensured that system (10), (15) is closed with respect to $\mathbf{P}$ and $w$.

However such a "simplification" entails consequences. Here, as always, the general principle operates: any generalization of a theory with a reduction in the axiomatic base ("entities") entails the inevitable complication of descriptive means in this case the mathematical apparatus. The generalization here refers to the reference to the general physical categories - energy and momentum obeying global conservation laws.

Indeed the above derivation of the basic equations shows that they are valid in the most compact (canonical) form only in a special local basis. Thus, we must consider all differential operations in an orthogonal curvilinear coordinate system defined by basis (10).

To emphasize this circumstance, we write the system (10), (15) in the following form: 


$$
\begin{aligned}
& \nabla^{\prime} \cdot \mathbf{T}+\frac{w}{\varepsilon(1+\varepsilon)} \nabla^{\prime} \varepsilon=\frac{\partial \mathbf{P}}{\partial t} \\
& \nabla^{\prime} \cdot \mathbf{P}-\frac{\nabla^{\prime} \varepsilon}{\varepsilon} \mathbf{P}=-\frac{\varepsilon}{c^{2}} \frac{\partial w}{\partial t} .
\end{aligned}
$$

Here the stroke means differentiation in the basis (12).

System (16) with external formal simplicity cannot be directly used to calculate the momentum and field energy. In fact to solve such a problem it is necessary to have explicit expressions for the transition from the local coordinate system defined by basis (12) to the laboratory coordinate system in which the experiment is fixed. In other words, geometry remains unknown.

Now we use the condition of local or thogonality of the field and the condition of amplitude coupling. This makes it possible to establish a connection between the geometry of the field and the geometry of the experiment, which is our further goal.

The problem is divided into two stages. First, we will find expressions for the divergence and gradient in the field basis through the Cartesian basis and the additional characteristic is the local rotation of the basis. Then we calculate the explicit form of the rotation angle vector.

\section{CONCLUSION OF DIFFERENTIAL OPERATIONS $\nabla^{\prime} \cdot \mathbf{P}, \nabla^{\prime} \cdot \mathrm{T}, \nabla^{\prime} \varepsilon$}

First of all it is necessary to introduce a Cartesian basis, which will correspond to the geometry of the propagation of a plane wave in a crystal as a continuum, when the response of the medium to an external action reduces to the material equation $D$ $=\varepsilon E=\left(1+\chi_{0}\right) E$. It is natural to take this state as the initial state, in relation to which, in the process of interaction of the X-ray wave with the crystal, a local variation of the basis $\mathbf{e}_{\mathrm{m}}$ occurs. Thus, we will consider the scattering problem in a special orthonormal basis ik defined by the vectors $\mathbf{D}_{0}, \mathbf{H}_{0}$, $\mathbf{P}_{0}$ in the continuous approximation:

$$
\mathbf{i}_{1}=\frac{\mathbf{D}_{0}}{D_{0}} ; \mathbf{i}_{2}=\frac{\mathbf{H}_{0}}{H_{0}} ; \mathbf{i}_{3}=\frac{\mathbf{P}_{0}}{P_{0}} .
$$

This means that all differential operators must be expressed precisely in this basis.

\subsection{Calculation $\nabla^{\prime} \cdot \mathbf{P}$}

We find $\nabla \cdot \mathbf{P}$ in the basis $\mathbf{i}_{k}$. The basic idea of the calculation is as follows. From the vector $\mathbf{P}(\mathbf{r})$ a second rank tensor can be constructed $\partial \mathbf{P} / \partial \mathbf{r}$ - a derivative with respect to the direction. By definition $\quad \nabla \cdot \mathbf{P}$ is a trace of a tensor $\partial \mathbf{P} / \partial \mathbf{r}$ as one of its invariants. We obtain $\partial \mathbf{P} / \partial \mathbf{r}$ in coordinateless form, using the so-called a Gâteaux derivative, or a weak derivative. This concept is a tool for nonlinear functional analysis, which, in particular, includes the classical calculus of variations [13, 14]. The weak derivative is defined in terms of a Gâteaux differential (a weak differential), which is introduced as the limit as $t$ $\rightarrow 0$ of the mapping of one normalized space $X$ to another $Y$ at an increment $x+$ th of an element of the space $X$.

Using the representation $\mathbf{P}=\mathrm{P}^{\mathrm{m}} \mathbf{e}_{\mathrm{m}}$, we obtain:

$$
\frac{\partial \mathbf{P}}{\partial \mathbf{r}}=P^{m} \frac{\partial \mathbf{e}_{m}}{\partial \mathbf{r}}+\left(\frac{\partial P^{m}}{\partial \mathbf{r}} \cdot \mathbf{i}_{s}\right) \cdot\left(\mathbf{e}_{m} \mathbf{i}_{s}\right) .
$$

The base $\mathbf{e}_{\mathrm{m}}$ is orthogonal at each point of $\mathbf{r}$, so it should be obtained by rotating the base $\mathbf{i}_{\mathrm{m}}$ around a certain axis at a small angle $\varphi$. It is clear that if the polarizability $\chi(\mathbf{r})$ is constant, then the angle $\varphi$ does not change. The smallness of the angle $\varphi$ is due to the small difference between the bases $\mathbf{e}_{\mathrm{m}}$ and $\mathbf{i}_{\mathrm{m}}$ by the variable component $\chi(\mathbf{r})$.

Thus a key concept is introduced into the theory - the local angle of rotation of the basis $\mathbf{e}_{\mathrm{m}}$. In the case of an ideal crystal, the angle $\varphi$ is determined by the three-dimensionally periodic polarizability $\chi(\mathbf{r})$ as a characteristic of the medium.

Introducing the vector $\varphi=\varphi \mathbf{k}$, where $\mathbf{k}$ is a unit vector along the axis of rotation, for small $\varphi$ from the Euler theorem [14] we obtain the law of the basis transformation $\mathbf{e}_{\mathrm{m}}$ :

$\mathrm{e}_{\mathrm{m}}=\mathrm{i}_{\mathrm{m}}+\varphi \times \mathbf{i}$.

Obviously, due to the locality of the basis $\mathbf{e}_{\mathrm{m}}$, the vector $\varphi$ is a local characteristic of the medium (field variable $): \varphi=\varphi(\mathbf{r})$.

Carrying out the corresponding calculations, we obtain the final expression for the momentum divergence:

$$
\nabla^{\prime} \cdot \mathbf{P}=\nabla \cdot \mathbf{P}-\nabla \cdot(\boldsymbol{\varphi} \times \mathbf{P}) .
$$




\subsection{Calculation $\nabla^{\prime} \cdot \varepsilon$}

We find the expression for the gradient $\varepsilon$. We will consider the transition from the basis $\mathbf{e}_{\mathrm{m}}$ to the basis $\mathbf{i}_{\mathrm{m}}$ as a substitute for the variable of the scalar function of the vector argument. With regard to connection

$$
\mathbf{r}^{\prime}=\mathbf{r}+\varphi \times \mathbf{r},
$$

using the Gâteaux derivative, we find:

$$
\begin{aligned}
& \frac{\partial \mathbf{r}^{\prime}}{\partial \mathbf{r}}=\mathbf{I}+\boldsymbol{\varphi} \times \mathbf{I}+\frac{\partial \boldsymbol{\varphi}}{\partial \mathbf{r}} \times \mathbf{r}, \\
& \left(\frac{\partial \mathbf{r}^{\prime}}{\partial \mathbf{r}}\right)^{T}=\nabla \mathbf{r}^{\prime}=\mathbf{I}-\mathbf{I}+\boldsymbol{\varphi}-\mathbf{r} \times\left(\frac{\partial \boldsymbol{\varphi}}{\partial \mathbf{r}}\right)^{T}= \\
& =\mathbf{I}-\mathbf{I} \times \boldsymbol{\varphi}-\mathbf{r} \times \nabla \boldsymbol{\varphi} .
\end{aligned}
$$

Here the sign $T$ means transposition.

Then the gradient of $\varepsilon$ is equal to:

$$
\nabla \varepsilon=(\mathbf{I}-\mathbf{I} \times \boldsymbol{\varphi}-\mathbf{r} \times \nabla \boldsymbol{\varphi}) \cdot \nabla^{\prime} \varepsilon .
$$

As a result, taking into account the smallness of $\varphi$, inversing the operator $\mathbf{I}-\mathbf{I} \times \boldsymbol{\varphi}-\mathbf{r} \times \nabla \boldsymbol{\varphi}$, we get

$$
\nabla^{\prime} \varepsilon=(\mathbf{I}+\mathbf{I} \times \boldsymbol{\varphi}+\mathbf{r} \times \nabla \varphi) \cdot \nabla \varepsilon .
$$

\subsection{Calculation $\nabla^{\prime} \cdot \mathbf{T}$}

To calculate this we use the formula for the divergence of the dyad:

$$
\nabla \cdot(\mathbf{u v})=(\nabla \cdot \mathbf{u}) \mathbf{v}+\mathbf{u} \cdot(\nabla \mathbf{v})
$$

as well as well-known vector analysis formulas. We get:

$$
\begin{aligned}
& \nabla^{\prime} \cdot \mathbf{T}=-\left(\nabla w \cdot \mathbf{e}_{3}\right) \mathbf{e}_{3}-\left(w \nabla \cdot \mathbf{e}_{3}\right) \mathbf{e}_{3}- \\
& -\left((\nabla \times \varphi) \cdot w \mathbf{e}_{3}\right) \mathbf{e}_{3}+\left(\left(\nabla \times w \mathbf{e}_{3}\right) \cdot \varphi\right) \mathbf{e}_{3}-w \mathbf{e}_{3} \cdot\left(\nabla^{\prime} \mathbf{e}_{3}\right) .
\end{aligned}
$$

Using the Gâteaux derivative, in the first approximation in $\varphi$, we get:

$$
\begin{aligned}
& \nabla^{\prime} \cdot \mathbf{T}=-\left(\nabla w \cdot \mathbf{i}_{3}\right)\left(\mathbf{i}_{3}+\boldsymbol{\varphi} \times \mathbf{i}_{3}\right)- \\
& -2 w\left(\nabla \cdot\left(\boldsymbol{\varphi} \times \mathbf{i}_{3}\right)\right) \mathbf{i}_{3} .
\end{aligned}
$$

\section{THE CALCULATION OF THE ROTATION VECTOR $\Phi$}

In order to explicitly take into account the locality of the basis, and therefore the dependence on $\chi(\mathbf{r})$ we turn to the orthogonal basis $\mathbf{m}_{i}$. that is not normalized to one. We select the new normalization from the requirement that when passing to the continual approximation $\left(\chi(\mathbf{r})=\chi_{0}=\right.$ const, respectively, $\varepsilon(\mathbf{r})=\varepsilon_{0}=$ const) the vectors $\mathbf{m}_{j}$ pass into $\mathbf{e}_{j}$. This takes into account the refraction of a wave in a crystal:

$$
\begin{aligned}
& \mathbf{m}_{1}=\frac{\mathbf{D}}{D_{0}}=\frac{\varepsilon \mathbf{E}}{\varepsilon_{0} E}=\frac{\varepsilon}{\varepsilon_{0}} \mathbf{e}_{1}, \\
& \mathbf{m}_{2}=\frac{\mathbf{H}}{H_{0}}=\frac{\sqrt{\varepsilon} \mathbf{E}}{\sqrt{\varepsilon_{0}} E}=\sqrt{\frac{\varepsilon}{\varepsilon_{0}}} \mathbf{e}_{2}, \\
& \mathbf{m}_{3}=\frac{\mathbf{P}}{P_{0}}=\frac{\sqrt{\varepsilon}|\mathbf{E}|^{2}}{\sqrt{\varepsilon_{0}}|E|^{2}} \mathbf{e}_{3}=\sqrt{\frac{\varepsilon}{\varepsilon_{0}}} \mathbf{e}_{3} .
\end{aligned}
$$

In this basis, the tensor $\mathbf{T}$ has the form

$$
\mathbf{T}=-w\left(\mathbf{e}_{3} \mathbf{e}_{3}\right)=-w \frac{\varepsilon}{\varepsilon_{0}}\left(\mathbf{m}_{3} \mathbf{m}_{3}\right)
$$

Lamé coefficients [15] for the basis mj are equal

$$
h_{1}=\left|\mathbf{m}_{1}\right|=\frac{\varepsilon}{\varepsilon_{0}} ; h_{2}=\left|\mathbf{m}_{2}\right|=\sqrt{\frac{\varepsilon}{\varepsilon_{0}}} ; h_{3}=\left|\mathbf{m}_{3}\right|=\sqrt{\frac{\varepsilon}{\varepsilon_{0}}} .
$$

Let us determine the microscopic displacement vector $\mathbf{u}=\mathbf{m}_{\mathbf{j}}-\mathbf{e}_{\mathbf{j}}$ during the transition from one basis to another along the corresponding directions of the axes of coordinates. Note that the vector $\mathbf{u}$ introduced by us, unlike the displacement vector of atomic planes in the formalism of the dynamic theory of X-ray diffraction for imperfect crystals [24] is not a macroscopically averaged value used in the continuum theory of elasticity [15]. Our microscopic displacement vector $\mathbf{u}$, due to locality $\chi(\mathbf{r})$ changes in the unit cell scale.

In this case we assume that $\varepsilon(\mathbf{r})=\varepsilon(\mathbf{h r})$ where $\mathbf{h}$ is the reciprocal lattice vector. Thus we actually use the model of an ideal crystal. Note that this restriction is by no means fundamental. If necessary you can enter all sorts of deviations from the ideal periodicity, i.e. to carry out a generalization of the theory on the deformed state of the crystal. For definiteness we choose the standard form of polarizability used in the dynamic theory of diffraction:

$$
\varepsilon=1+\chi_{0}+\chi_{h}\left(\exp (i \mathbf{h r} \mathbf{r})+\frac{\chi_{\bar{h}}}{\chi_{h}} \exp (-i \mathbf{h r} \mathbf{r})\right)=
$$

$$
=\varepsilon_{0}+\chi_{h} f(\mathbf{h r}) \text {. }
$$

Then for the microscopic displacement vector $u$, we have:

$$
\mathbf{u}=\left(h_{j}-1\right) \mathbf{e}_{j}=\left(h_{j}-1\right)\left(\mathbf{i}_{j}+\varphi \times \mathbf{i}_{j}\right) .
$$

Introduction of the vector $\mathbf{u}$ actually means using an alternative concept of describing the interaction of a field with a substance. In the usual description, we use the representation of interaction unfolding in Euclidean space with invariable metric 
characteristics. The space in this concept plays the passive role of the "container" of events.

An alternative concept gives the theory a geometric interpretation, which goes back to Einstein's idea that the geometry of space is not given ad hoc but is determined by the interaction in this case, the field and matter. Thus, the geometry acquires a dynamic nature [6].

In this case the response of the medium to external disturbance is considered as a local change in the geometry - a rotation of the base determined by the structural characteristics of the medium. Or otherwise the interaction changes the geometry.

Next we use the results of the linear theory of elasticity [16], in which small displacements of the medium are considered as field variables determining the symmetric deformation tensor $\boldsymbol{\varepsilon}(\mathbf{r})$ and the antisymmetric rotation tensor $\omega(\mathbf{r})$. As is known any antisymmetric tensor is associated with its dual vector. In the case of $\boldsymbol{\omega}(\mathbf{r})$ this vector is the axial vector of small rotations $\varphi$. The geometric meaning of the vector $\varphi$ is to rotate a neighborhood of a given point of the medium as a whole around the axis of rotation and the angle and direction of rotation coincide respectively with the length and direction of the vector $\varphi$.

Based on this interpretation, the following fundamental relation is derived in the theory of elasticity which determines the rotation vector $\varphi$ through the rotor of the displacement vector [16]:

$$
\boldsymbol{\varphi}=\frac{1}{2} \nabla \times \mathbf{u} \text {. }
$$

According to (22) using (21) we get:

$$
\begin{aligned}
& 2 \boldsymbol{\varphi}=\nabla \times \mathbf{u}=\nabla \times\left(h_{j}-1\right)\left(\mathbf{i}_{j}+\boldsymbol{\varphi} \times \mathbf{i}_{j}\right)= \\
& =\nabla\left(h_{j}-1\right) \times \mathbf{i}_{j}+\nabla \times\left(\left(h_{j}-1\right)\left(\boldsymbol{\varphi} \times \mathbf{i}_{j}\right)\right) .
\end{aligned}
$$

Or, taking into account (20) and the smallness of $\chi$ :

$$
\begin{aligned}
& 2 \boldsymbol{\varphi}=\frac{\chi_{h}}{2} \nabla f \times\left(2 \mathbf{i}_{1}+\mathbf{i}_{2}+\mathbf{i}_{3}\right)+ \\
& +\frac{\chi_{h}}{2} \nabla \times\left(f\left(2 \boldsymbol{\varphi} \times \mathbf{i}_{1}+\boldsymbol{\varphi} \times \mathbf{i}_{2}+\boldsymbol{\varphi} \times \mathbf{i}_{3}\right) .\right.
\end{aligned}
$$

We look for the solution of equation (23) in the form of a series expansion in a small parameter $\chi_{h}$ : $\varphi=\varphi_{0}+\chi_{\mathrm{h}} \varphi_{1}+\ldots$

Acting by the standard perturbation theory method, we obtain the solution in the zero approximation $\varphi_{0}=0$ and the first order approximation:

$$
\boldsymbol{\varphi}_{1}=\frac{1}{4}\left(\nabla f \times\left(2 \mathbf{i}_{1}+\mathbf{i}_{2}+\mathbf{i}_{3}\right)\right) .
$$

Then the solution of equation (23) in the first order approximation takes the form:

$$
\boldsymbol{\varphi}=\frac{\nabla \chi}{4} \times\left(2 \mathbf{i}_{1}+\mathbf{i}_{2}+\mathbf{i}_{3}\right) .
$$

According to (24), the value of $\varphi$ is a local measure of the deviation of the geometric characteristics of the medium with respect to the propagation of electromagnetic waves from the continual approximation.

The vector $\varphi$ under the condition $\chi=\chi(\mathbf{h r})$ i.e. if the crystal is perfect, it retains its orientation in space and does not coincide with the basis vectors $\mathbf{i}_{\mathrm{m}}$. If it coincided with $\mathbf{i}_{1}$, then $\sigma$-polarization would be realized, and if with $\mathbf{i}_{2}-\pi$-polarization. Consequently, with the transmission of a wave a superposition of $\sigma$ - and $\pi$-polarizations is realized and this is apparently a general result. Hence the conclusion: the separation of waves by polarization when considering problems of X-ray wave scattering on a crystal is not quite correct even in the case of an ideal crystal.

Of course, the conservation of the orientation of the vector $\varphi$ in space is a specific property of only an ideal crystal. In other cases say when taking into account the deformation of the crystal the vector $\varphi$ will locally change its orientation in space, for example, precess around the original direction corresponding to the ideal crystal.

\section{CONCLUSION OF THE BASIC EQUATION SYSTEM IN THE CARTESIAN BASIS}

Linearize the fundamental system (16) by a small quantity $\chi$ :

$$
\begin{aligned}
& \nabla^{\prime} \cdot \mathbf{T}+w \nabla \varepsilon=\frac{\partial \mathbf{P}}{\partial t}, \\
& \nabla^{\prime} \cdot \mathbf{P}-\nabla \varepsilon \mathbf{P}=-\frac{\varepsilon}{c^{2}} \frac{\partial w}{\partial t} .
\end{aligned}
$$

Then using explicit expressions for divergences and gradient (17-19), we obtain: 


$$
\begin{aligned}
& -\left(\nabla w \cdot \mathbf{i}_{3}\right)\left(\mathbf{i}_{3}+\boldsymbol{\varphi}+\mathbf{i}_{3}\right)-2 w\left(\nabla \cdot\left(\boldsymbol{\varphi} \times \mathbf{i}_{3}\right)\right) \mathbf{i}_{3}+ \\
& +w \nabla \chi=\frac{\partial \mathbf{P}}{\partial t}, \\
& \nabla \cdot \mathbf{P}-\nabla \cdot(\boldsymbol{\varphi} \times \mathbf{P})-\nabla \chi \cdot \mathbf{P}=-\frac{1+\chi(\mathbf{r})}{c^{2}} \frac{\partial w}{\partial t} .
\end{aligned}
$$

These relations represent the fundamental equations of the above theory, which allow one to calculate the momentum and energy of a field when it interacts with a crystal. As in the usual dynamic theory of diffraction (say, the Takagi equations), such an interaction is parametric in nature, which means that a parametric resonance should be observed under certain geometric conditions. Of course one should expect that these conditions correspond to the Laue equation, and the resonance physically corresponds to the appearance of a diffraction wave (in terms of this theory the appearance of the corresponding component of the total momentum).

Equations (25) in contrast to the diffraction equations for fields depend parametrically not only on $\chi$, but on $\nabla \chi \sim \mathbf{h}$. Note that the equations are linear which makes it possible to use the principle of superposition when constructing the solution.

\section{CONCLUSION}

Let us outline the further development of the theory as applied to the description of diffraction scattering in a crystal. The basic equations (25) obviously cannot be solved exactly therefore it is necessary to use the methods of perturbation theory. In this case, as a small parameter, as in the theory $[3,4]$, the Fourier component of polarizability $\chi_{h}$ is used which is associated with diffraction scattering. Unperturbed equations (zero approximation)

$$
\begin{aligned}
& -\left(\nabla w_{0} \cdot \mathbf{i}_{3}\right) \mathbf{i}_{3}=\frac{\partial \mathbf{P}}{\partial t}, \\
& \nabla \cdot \mathbf{P}=-\frac{1+\chi_{0}}{c^{2}} \frac{\partial w_{0}}{\partial t}
\end{aligned}
$$

describe the momentum-energy propagation in a medium as in a continuum.

At the same time unlike the usual wave equations the physical solution for the zero approximation is formed not from harmonic waves but from their products. In fact, for harmonic waves, there is a dependence:

$$
\begin{aligned}
& \mathbf{P} \sim \mathbf{E} e^{I \psi} \times \mathbf{H} e^{I \psi} \sim E H e^{I 2 \psi} \\
& w \sim\left(E e^{I \psi}\right)^{2}+\left(H e^{I \psi}\right)^{2} \sim E H e^{I 2 \psi} .
\end{aligned}
$$

Then for $\mathbf{P}_{0}$ and $w_{0}$, functionally invariant solutions in the form of squares of harmonic waves containing constant (non-oscillating) components should be chosen. Obviously it is these components that are fixed in the experiment. Such solutions are not harmonic therefore it is impossible to get rid of time derivatives and go to a purely spatial problem by simple substitution. Consequently, it is necessary to develop a perturbation theory for a system of partial differential equations of the type (26).

This problem is the subject of further research.

\section{ACKNOWLEDGMENT}

The author expresses deep gratitude to Professor Yu.P. Khapachev for a number of valuable comments on the merits and useful discussion.

\section{REFERENCES}

1. James RW. The Optical Principles Of The Diffraction Of X Rays. Vol II. London, G. Bell and sons Ltd. 1962, $664 \mathrm{p}$.

2. Pinsker ZG. X-ray crystal optics. Moscow, Nauka Publ., 1982, 392 p.

3. Dyshekov AA, Khapachev YuP. New analytical approaches to the problems of X-ray diffraction crystal optics. Nalchik, Kabardino-Balkarian State. university Publ., 2010, 45 p.

4. Dyshekov AA. Generalization of the nonstandard approach in the dynamic theory of diffraction for deformed crystals. Crystallography Reports, 2013, 58(7):984-989.

5. Polak LS (ed.). Variational principles of mechanics. Collection of articles of the classics of science. Moscow, Fizmatlit Publ., 1959, 932 p.

6. Konopleva NP, Popov VN. Gange fields. Moscow, Atomizdat Publ., 1972, 240 p.

7. Katanaev MO. Geometric methods in mathematical physics. Moscow-Kazan, Steklov Math.Inst- Kaz. Fed. Univ. Publ., 2016, 1570 p. https://arxiv. org/pdf/1311.0733 (in Russian).

8. Lourtioz J.-M., Benisty H., Berger V., Gerard J.M, Maystre D., Tchelnokov A. Photonic crystals. Towards Nanoscale Photonic Devices. SpringerVerlag Berlin Heidelberg, 2008, 514 p.; DOI: 10.1007/978-3-540-78347-3. 
9. Aristov VV, Shabelnikov LG. Modern advances in X-ray refractive optics. UFN, 178(1):61-83 (in Russ.).

10. Novozhilov YuV, Yappa YuA. Electrodynamics. Moscow, Nauka Publ., 1978, 352 p.

11. Fedorov FI. Theory of gyrotropy. Minsk, Science and technology Publ., 1976, 456 p.

12. Dubrovin BA, Novikov SP, Fomenko AT. Modern geometry. Methods and applications. Moscow, Nauka Publ., 1986, 760 p.

13. Kolmogorov AN, Fomin SV. Elements of the theory of functions and functional analysis. Moscow, Nauka Publ., 1981, 544 p.

14. Vilchevskaya EN. Tensor algebra and tensor analysis. Tutorial. St. Petersburg, SPb State Technical University Publ., 2012, 45 p.

15. Lurie AI. Theory of elasticity. Moscow, Nauka Publ., 1970, 940 p.

16. Sirotin YuI, Shaskolskaya MP. Fundamentals of crystal physics. Moscow, Nauka Publ., 1975. 680 p. 